\title{
USO DE LA PLATAFORMA MOODLE COMO HERRAMIENTA PARA LA EVALUACIÓN CONTINUA DE ESTUDIANTES EN EL ESPACIO EUROPEO DE EDUCACIÓN SUPERIOR
}

Isidoro Iván Cuesta-Segura1: Universidad de Burgos. España iicuesta@ubu.es

Jesús Manuel Alegre-Calderón: Universidad de Burgos. España iicuesta@ubu.es

\section{RESUMEN}

Uno de los pilares fundamentales en los que se sustenta el Espacio Europeo de Educación Superior (EEES) es la evaluación y seguimiento del trabajo del alumnado de forma continuada a lo largo del curso. En este sentido los entornos de trabajo que proporciona la Web 2.0 se presentan como una herramienta realmente útil a la hora de abordar el reto de este tipo de evaluación. La Universidad de Burgos implantó un nuevo campus virtual, basado en Moodle, en el curso 2009-2010 para las nuevas titulaciones de Grado y Master. Moodle cuenta con gran cantidad de módulos y de actividades que pueden ser evaluadas por el profesorado, y que sirven para otorgar una calificación al estudiante. Entre estos se cuenta con el módulo de cuestionarios, el cual va a permitir al personal docente diseñar sus propios cuestionarios y aplicarlos a sus estudiantes de forma on-line. Este trabajo presenta la aplicación de cuestionarios en Moodle y los diferentes tipos de preguntas disponibles para generarlos como herramienta para la evaluación continua.

PALABRAS CLAVE: Evaluación continua - Web 2.0 - Moodle - Módulo de Cuestionarios

\footnotetext{
1 Autor correspondiente

Isidoro Iván Cuesta-Segura: Doctor. Profesor Ayudante. Universidad de Burgos. Burgos (España).

Correo: $\underline{\text { iicuesta@ubu.es }}$
} 


\title{
USING MOODLE PLATFORM AS A TOOL FOR THE CONTINUOUS ASSESSMENT OF STUDENTS IN EUROPEAN HIGHER EDUCATION AREA
}

\begin{abstract}
One of the main changes European Higher Education Area (EHEA) is related to the continuous assessment system of the student work throughout the course. In this sense, the work environment that provides Web 2.0 is presented as a really useful tool to achieve this kind of continuous assessment. The University of Burgos introduced in 2009-2010 a new virtual campus system based on Moodle platform, for the new Bachelor and Master degrees. Moodle has a large number of modules and activities that can be used by teachers to assess the teaching-learning process. One of the most useful option in Moodle to evaluate a large group of students is the questionnaire module, which will allow the teacher to design a large number of individual questionnaires, one different for each student, that can be fill and corrected on-line. This paper presents this application for questionnaires in Moodle and the variety of options available to generate them as a tool for a continuous assessment of the students.
\end{abstract}

KEY WORDS: Continuous assessment system - Web 2.0 - Moodle - Questionnaire module

\section{INTRODUCCIÓN}

Uno de los pilares fundamentales en los que se sustenta el Espacio Europeo de Educación Superior (EEES) es la evaluación y seguimiento del trabajo del alumnado de forma continuada a lo largo del curso, es decir, se basa en la evaluación continua. Son indudables las ventajas que presenta este tipo de seguimiento para la adquisición de competencias, así como un mejor rendimiento y aprovechamiento del tiempo por parte de los alumnos/as. Sin embargo, este tipo de evaluación puede resultar especialmente laboriosa para el personal docente en aquellas asignaturas que cuenten con un alumnado muy numeroso.

En este sentido los entornos de trabajo que proporciona la Web 2.0 se presentan como una herramienta realmente útil a la hora de abordar el reto de la evaluación continuada en grupos numerosos. Estos entornos virtuales dotados de diferentes módulos permiten la interacción docente-alumnado de manera on-line mejorando de esta manera el aprovechamiento del tiempo por ambas partes. 
Con todo ello se busca conseguir una evaluación educativa (Wiggins, 1998), en primer lugar que esté diseñada para enseñar y, en segundo, que facilite la retroalimentación entre alumnado y personal docente. Ejemplos de estos entornos virtuales son The Rubric Builder (www.rubricbuilder.on.ca/), Rubistar (www.rubistar.4teachers.org), o incluso herramientas que se pueden integrar dentro del propio Moodle como EvalCOMIX (Ibarra, 2010).

Como consecuencia de las ventajas anteriores la Universidad de Burgos implantó un nuevo campus virtual (UBUVirtual, www.ubuvirtual.ubu.es), basado en Moodle, en el curso 2009-2010 para las nuevas titulaciones de Grado y Master. Moodle es un proyecto en continuo desarrollo, cuya base pedagógica subyacente se sitúa dentro del constructivismo social (colaboración, actividades...), pudiendo favorecer también el aprendizaje social y el aprendizaje activo (Ferdig, 2007).

Moodle cuenta con gran cantidad de módulos y de actividades que pueden ser evaluadas por el profesorado, y que servirán para otorgar una calificación al estudiante. Entre estos se cuenta con el módulo de cuestionarios, el cual va a permitir al personal docente diseñar sus propios cuestionarios y aplicarlos a sus estudiantes de forma on-line. Una de las ventajas de los cuestionarios es que las respuestas se corrigen y califican de forma automática, con lo que el ahorro de tiempo de corrección es importante, sobre todo cuando se cuenta con un gran número de alumnos/as.

A pesar de éstas y otras ventajas, no hay que olvidar que se pueden llegar a plantear ciertas dificultades y errores comunes en la aplicación de los cuestionarios, llegándose a afirmar que a menudo es muy difícil que el personal docente cree test con una elevada calidad debido a la ineficacia de los propios sistemas (Smoline, 2008).

\section{METODOLOGÍA}

La presente investigación se centra en las aplicaciones de Moodle del entorno UBUVirtual para favorecer la evaluación continua en grupos con un elevado número de alumnos/as. En particular, se presenta la aplicación de cuestionarios y los diferentes tipos de preguntas existentes para el desarrollo y evaluación de prácticas en grupos numerosos, con el objetivo de conseguir realizar de forma efectiva los $\mathrm{N}$ ejercicios diferentes por cada práctica, y que aparezcan de forma automática en el entorno de trabajo de cada alumno/a, posibilitando en última instancia la evaluación continua. 


\section{ANÁLISIS Y DISCUSIÓN}

\subsection{Evaluación continuada clásica}

Una de las formas más habituales de evaluación continuada consiste, por ejemplo, en el desarrollo y entrega de una serie de prácticas a lo largo del curso, lo que presenta dos inconvenientes. Por un lado, la tendencia natural del alumnado a optimizar su tiempo, copiando la práctica a otros compañeros, con lo cual perdemos el objetivo de la actividad práctica, que no es otro que la comprensión y aprendizaje de lo incluido en ella. Por otro lado, se debe evaluar el contenido de cada práctica individualmente, elevando de manera considerable el tiempo dedicado a la corrección de las mismas. Todo profesor/a que se haya enfrentado a este tipo de grupo conoce de las dificultades que entraña llevar a buen término un adecuado programa de prácticas, y que realmente se cumpla el objetivo buscado.

En consecuencia, para la efectiva realización de la evaluación continua, con un grupo numeroso, debemos conseguir dos objetivos:

a) asegurar la realización de trabajos, prácticas, etc... de forma individualizada (o en grupos reducidos), y

b) la corrección o evaluación de los mismos, de la forma más efectiva posible. Es aquí donde el uso de la aplicación de cuestionarios de Moodle cobra un especial interés.

\subsection{Generación de preguntas para cuestionarios en UBUVirtual}

La generación de cuestionarios en el entorno de trabajo de UBUVirtual puede ser más o menos compleja en función del tipo de preguntas que se deseen formular. Cada cuestionario estará formado por un conjunto de preguntas que el personal docente debe crear previamente en el Banco de preguntas de UBUVirtual, mostrado en la Figura 1.

También existe la posibilidad de importar preguntas desde diferentes formatos de fichero (GIFF, Examview, Moodle XML, etc.), accediendo a ello a través de la pestaña "Importar" que se observa en la Figura 1. 


\section{Banco de preguntas}

\begin{tabular}{|c|c|c|c|c|}
\hline \multicolumn{5}{|c|}{ Categoría Tema 3 Diagramas (4) } \\
\hline \multicolumn{5}{|c|}{$\square$ Incluir sub-categorías } \\
\hline \multicolumn{5}{|c|}{$\square$ Mostrar también preguntas antiguas } \\
\hline \multicolumn{5}{|c|}{$\square$ Mostrar el texto de la pregunta en la lista de preguntas } \\
\hline \multirow{2}{*}{\multicolumn{2}{|c|}{ Crear una nueva pregunta }} & Escoger... & & $?$ \\
\hline & & Escoger... & & \\
\hline \multirow{8}{*}{\multicolumn{4}{|c|}{ 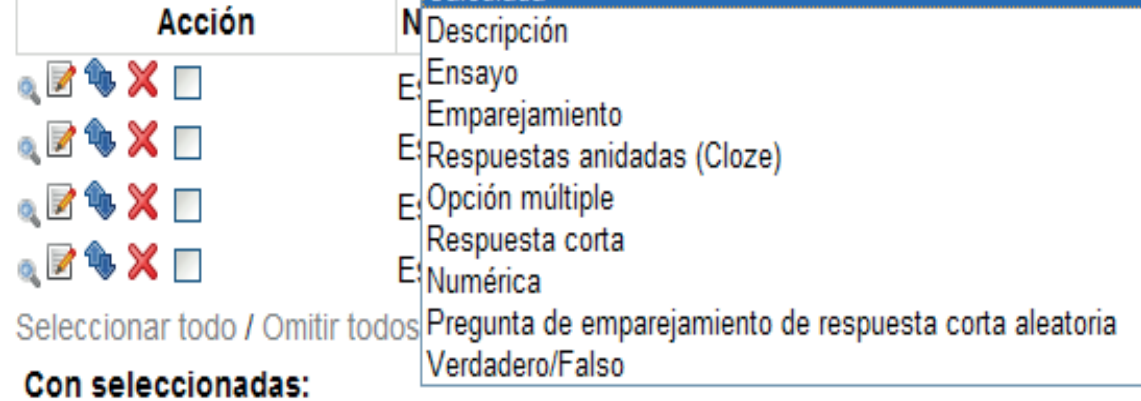 }} & Escriba \\
\hline & & & & $\stackrel{2+2}{=}$ \\
\hline & & & & $\stackrel{2+2}{=} \stackrel{2}{?}$ \\
\hline & & & & $\begin{array}{cc}-1 \\
2+2\end{array}$ \\
\hline & & & & \\
\hline & & & & 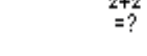 \\
\hline & & & & \\
\hline & & & & \\
\hline Borrar & Mover $a \gg$ & na 3 Diagramas (4) & & $v$ \\
\hline
\end{tabular}

Figura 1. Banco de preguntas en UBUVirtualFuente: Elaboración propia

Los posibles tipos de preguntas que se pueden crear en el entorno de trabajo son las que se describen a continuación:

\section{Opción múltiple}

Para responder a este tipo de pregunta el alumnado debe escoger entre múltiples respuestas. Hay dos tipos de preguntas de opción múltiple: las de respuesta única y las de respuesta múltiple.

Las preguntas de respuesta única permiten escoger sólo una respuesta, generalmente todas las calificaciones para este tipo de pregunta deberían ser positivas. Las preguntas de respuesta múltiple permiten escoger una o más respuestas. Cada respuesta puede tener una puntuación positiva o negativa, de tal forma que si se escogen TODAS las opciones no se obtenga necesariamente una buena calificación. Si la puntuación total es negativa, la calificación total de esta pregunta será cero. En este tipo de preguntas hay que tener especial cuidado, ya que es posible crear preguntas que tengan puntuaciones superiores al 100\%.

Finalmente, cada respuesta (correcta o incorrecta) debe incluir la corrección. Si se desea esta corrección se puede mostrar al alumnado al lado de cada una de sus 


\section{Respuesta corta}

Para responder a este tipo de pregunta el alumnado escribe una palabra o frase. Hay varias posibles respuestas correctas, con diferentes puntuaciones. Las respuestas pueden o no ser sensibles a las mayúsculas, es decir, si se selecciona la opción "Sensible a las mayúsculas", se podrán tener puntuaciones diferentes para "Palabra" o "palabra".

\section{Numérica}

Desde el punto de vista del alumnado, una pregunta numérica es muy semejante a una pregunta de respuesta corta. La diferencia es que la pregunta numérica es un número y permite un margen de error.

Esto permite habilitar un rango continuo de posibles respuestas acertadas, es decir, si la respuesta correcta es 30, y el margen aceptable es 5, cualquier número entre 25 y 35 se aceptará como correcto.

\section{Verdadero / Falso}

Para responder a este tipo de pregunta el alumno/a tiene que elegir entre dos opciones: Verdadero o Falso.

\section{Emparejamiento}

Presenta una lista de preguntas junto con una lista de respuestas, se debe seleccionar la respuesta correcta que corresponda a cada pregunta de la lista.

\section{Preguntas incrustadas (Cloze)}

Este tipo de pregunta es muy flexible y semejante al popular formato conocido como "Cloze". Consiste en un texto que puede integrar (en formato Moodle) varias preguntas de tipo opción múltiple, preguntas cortas y numéricas.

Las preguntas consisten en un pasaje de texto (en formato Moodle) que contiene varias respuestas incrustadas tales como respuestas de elección múltiple, respuestas cortas y respuestas numéricas.

Actualmente no se dispone de una interfaz gráfica para crear este tipo de preguntas, de modo que es preciso especificar el formato de la pregunta usando la caja de texto, o bien imbortarlas desde archivos externos. 
La diferencia es que las preguntas y respuestas son extraídas aleatoriamente del conjunto de preguntas de respuesta corta existentes en la categoría, por lo que cada intento hecho sobre un mismo examen tendrá diferentes preguntas y respuestas.

\begin{abstract}
Aleatoria
Las preguntas aleatorias son un tipo especial de pregunta. Cuando se pone una pregunta aleatoria en un cuestionario, entonces en cada intento se elegirá al azar una pregunta de la categoría completa. La calificación máxima de la pregunta será siempre la que se haya elegido como calificación para la pregunta aleatoria.

Si se genera un cuestionario con, por ejemplo, 10 preguntas aleatorias, cada estudiante tendrá un conjunto totalmente distinto de 10 preguntas cada vez que responda al cuestionario. Es posible mezclar preguntas aleatorias y no aleatorias si se desea que ciertas preguntas sean siempre incluidas.
\end{abstract}

\title{
Descripción
}

Este formato no es una pregunta en sentido estricto. Se limita a mostrar un texto o si se desea algún gráfico sin necesidad de responder. Puede ser útil, por ejemplo, para dar información previa antes de un grupo de preguntas.

\section{Calculadas}

Las preguntas calculadas ofrecen una forma de crear preguntas numéricas individuales por medio del uso de comodines que son sustituidos por los valores individuales cuando se responde el cuestionario.

\subsection{Metodología}

Para solventar los problemas expuestos anteriormente se ha desarrollado una metodología, basada en el uso de la aplicación de cuestionarios de Moodle, que permita la evaluación continua, así como ofrecer al alumnado unas prácticas de calidad en las que se consoliden y se pongan en uso los conocimientos adquiridos en la docencia teórica. 


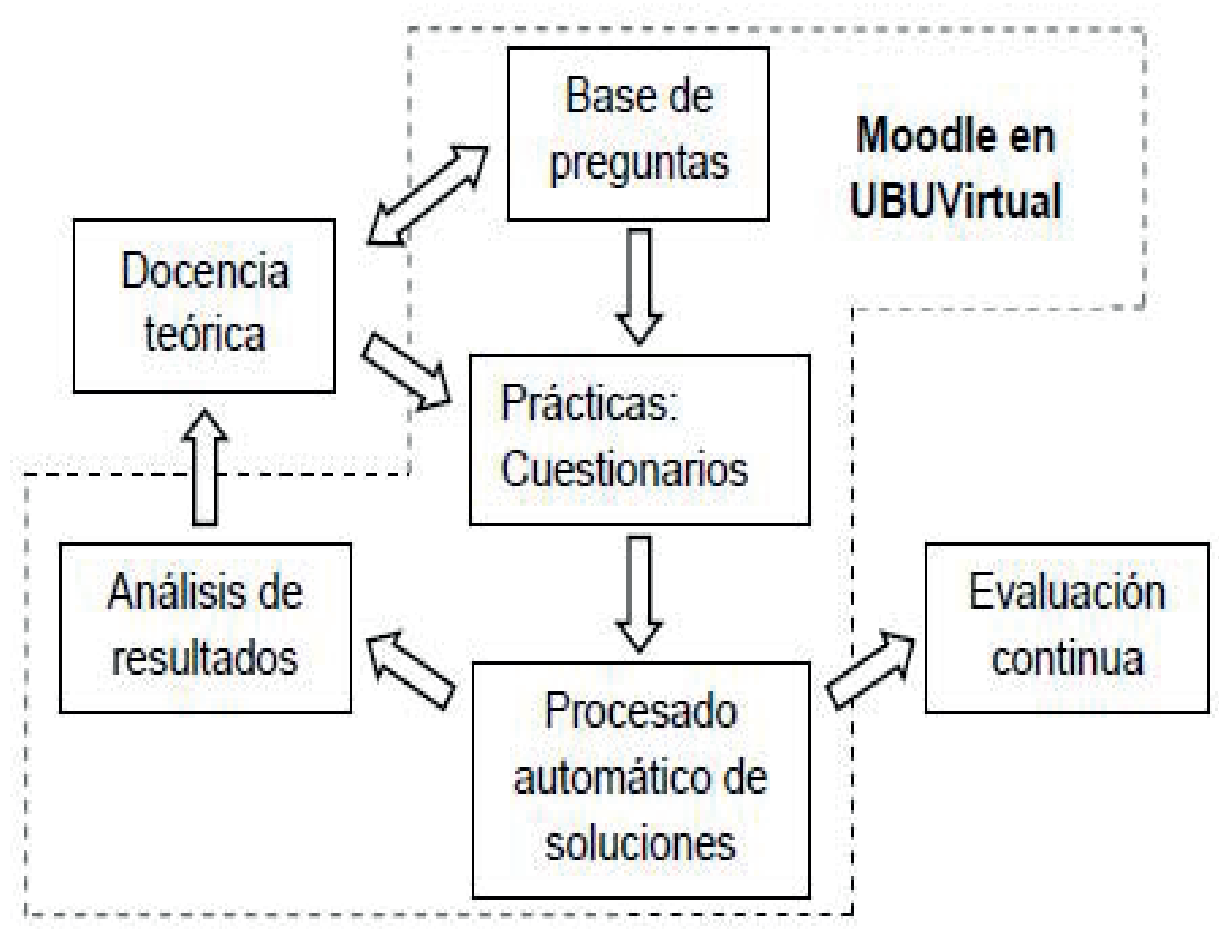

Figura 2. Esquema metodológico. Fuente: Elaboración propia

La Figura 2 muestra un esquema de la metodología desarrollada, en la que es necesario un trabajo previo por parte del personal docente, que tiene que generar y guardar on-line, por cada tema o tipo de problema, las preguntas que más adelante se utilizarán para los cuestionarios.

Una vez creadas las preguntas deseadas, la aplicación de cuestionarios de Moodle permite generar de manera aleatoria un cuestionario diferente para cada alumno/a, que tendría que ser contestado de manera on-line durante la realización de la práctica.

De esta manera se verán obligados a poner en uso los conocimientos adquiridos si desean completarlo con éxito. La posterior corrección de cada uno de los cuestionarios es realizada de forma automática por la aplicación de cuestionarios de Moodle, reduciendo al mínimo el tiempo que tendría que emplear el profesorado para evaluar cada uno de los cuestionarios. 


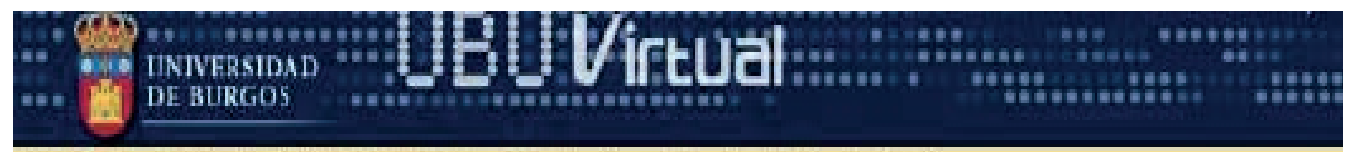

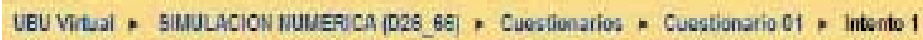

\title{
Cuestionario 01 - Intento 1
}

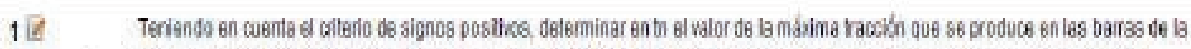

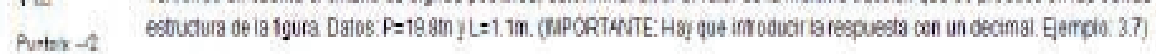

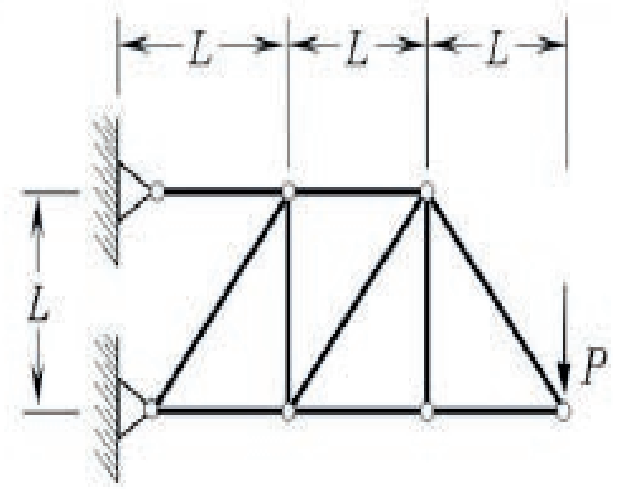

Respusest:

Eniar.

218 Teriendo an cuanta el citario de signas posthos, determinar an to al esfuerzo normal an la barra AB do la figura. Dabse. P=4 3th y Puntax $\rightarrow 3$ L=1.0m (IMPORTNWTE Hay que introdudr la respuesta con un dedmal. Ejemplo. 3.T)

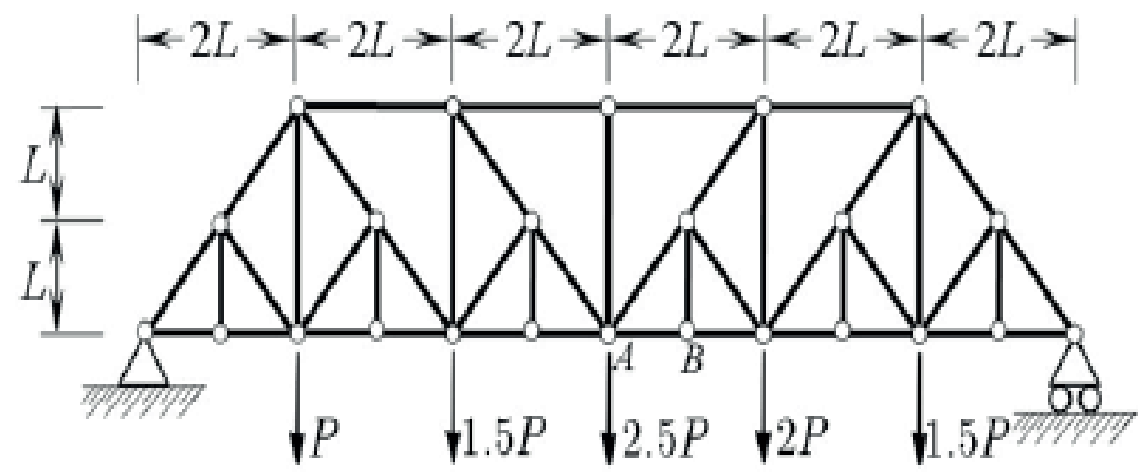

Respuests:

Enviar 
La evaluación de cada alumno/a puede consultarse en cualquier instante (Figura 4), permitiendo conocer en tiempo real los logros obtenidos de manera individual o por el grupo. Este tiempo ganado será empleado por parte del personal docente en analizar los resultados obtenidos, identificando las fortalezas y debilidades de cada uno de los alumnos/as o del grupo en general. Para cada cuestionario existen diferentes opciones para analizarlos y extraer de ellos la información relevante para realizar y mejorar la evaluación continua (Figura 5).

Esta información será de gran utilidad para retroalimentar tanto los contenidos docentes como las preguntas de los cuestionarios, consiguiendo en última instancia una mejora continua de la enseñanza universitaria.

\section{Calificador}

\begin{tabular}{|c|c|c|c|c|}
\hline \multicolumn{5}{|l|}{ ELASTICIDAD Y RESISTENCIA .... } \\
\hline 図 Cuestionario 01 - Tema 2 ... & 国圈Cuestionario 02 Tema $2 \ldots$. & 揭Cuestionario 03 Tema 3 ... & 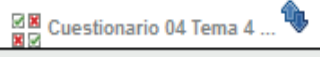 & 远 Total del curso \\
\hline 6,60 & 9,20 & 9,10 & - & 83,00 \\
\hline . & 9,10 & 9,20 & . & 91,50 \\
\hline . & . & - & - & \\
\hline 10,00 & 6,30 & 9,80 & . & 87,00 \\
\hline - & 10,00 & 9,20 & . & 96,00 \\
\hline . & 6,60 & 8,00 & . & 73,00 \\
\hline 3,00 & 9,70 & 10,00 & . & 75,67 \\
\hline 6,90 & 5,50 & 5,00 & . & 58,00 \\
\hline 10,00 & 1,60 & - & . & 58,00 \\
\hline . & 9,40 & 8,90 & . & 91,50 \\
\hline 9,10 & 5,20 & 5,00 & . & 64,33 \\
\hline
\end{tabular}

Figura 4. Ejemplo de consulta de calificaciones en UBUVirtual.

Fuente: Elaboración propia 


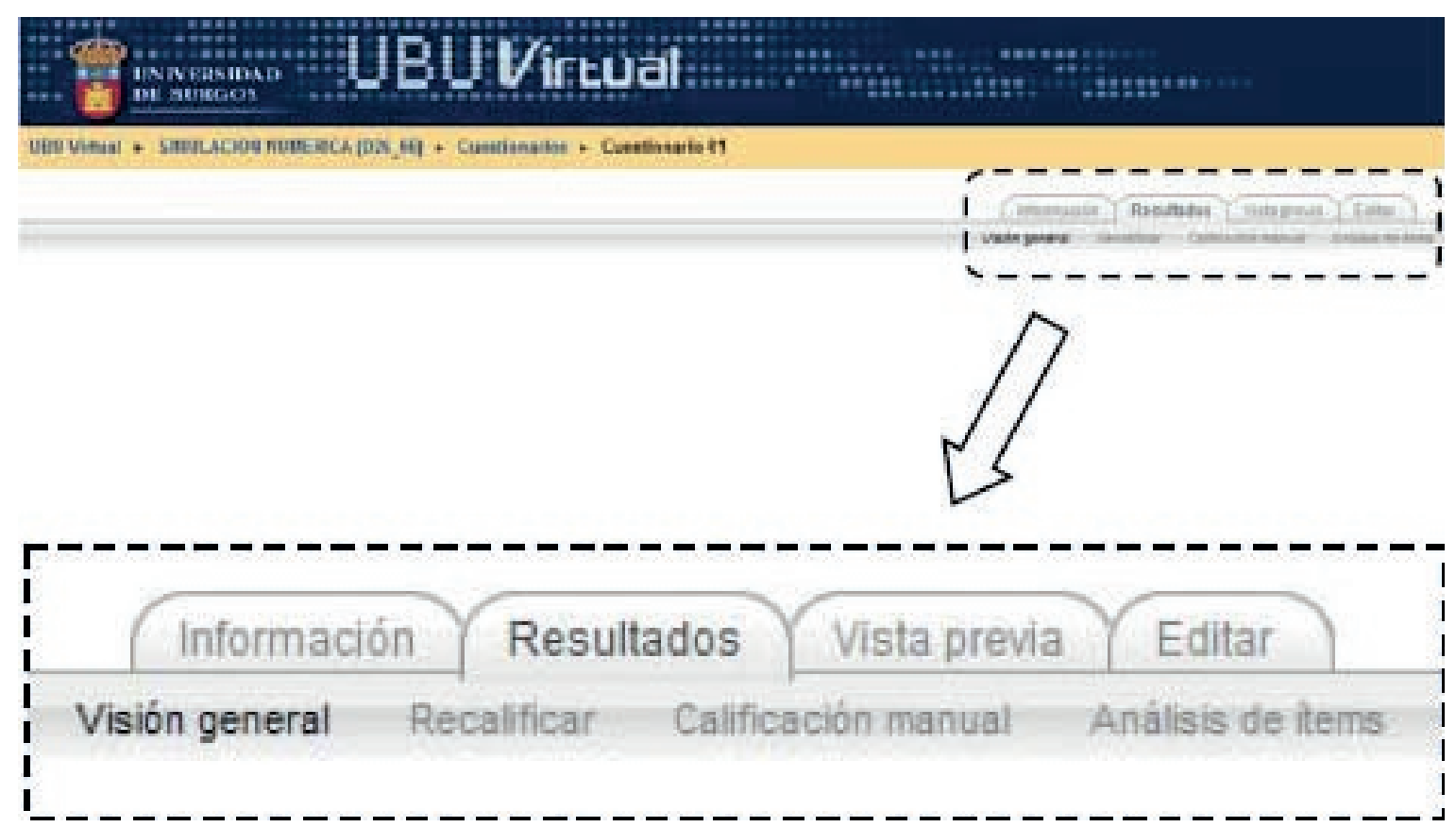

Figura 5. Resultados del cuestionario

Fuente: Elaboración propia

\section{CONCLUSIONES}

En el presente trabajo se ha puesto de manifiesto la gran utilidad de la aplicación de cuestionarios de Moodle, implementada en el entorno de trabajo UBUVirtual, para favorecer la evaluación continua en grupos numerosos, ya que permite implementar en cada cuestionario diferentes tipos de preguntas, las cuales podrán ser diferentes para cada uno de los alumnos/as consiguiendo así evitar el plagio de los cuestionarios.

Por otro lado, la evaluación automática de los cuestionarios permitirá disfrutar al docente de mayor tiempo para analizar los resultados obtenidos por el alumnado, de forma que tenga una base sólida para tomar la decisión de modificar los contenidos docentes, generar nuevas preguntas o hacer especial hincapié en algún contenido en particular, mejorando de esta forma la evaluación continuada.

\section{REFERENCIAS}

Ferdig, R. (2007). Examining social software in teacher education. Journal of Technology and Teacher Education, 15 (1): 5-10.

Ibarra, Ma S. et. Al. (2010). EvalCOMIX en Moodle: Un medio para para favorecer 
Wiggins, G. (1998). Educative Assessment. Designin assessments to inform and improve student performance. San Francisco: CA: Jossey-Bass.

\section{Isidoro Iván Cuesta Segura}

Profesor Ayudante Doctor en Mecánica de los Medios Continuos en la Escuela Politécnica Superior de la Universidad de Burgos. Es Ingeniero de Organización Industrial por la Universidad de Burgos, y Doctor por la misma Universidad en el campo de la simulación local de la fractura de materiales. Actualmente es miembro de la Sociedad Española de Integridad Estructural - Grupo Español de Fractura y del Grupo de Investigación de Integridad Estructural (GIE) de la Universidad de Burgos. Es autor de más de 10 publicaciones científicas incluidas en el JCR. Participa como investigador en varios proyectos de investigación del plan nacional, en proyectos regionales y en convenios de investigación con empresas del campo industrial.

\section{Jesús Manuel Alegre Calderón}

Profesor Titular de Universidad en Mecánica de los Medios Continuos en la Escuela Politécnica Superior de la Universidad de Burgos, dentro del Departamento de Ingeniería Civil. Es Ingeniero Industrial, dentro de la Especialidad de Mecánica y Materiales por la Universidad de Cantabria, y Doctor Ingeniero Industrial. Es Coordinador del Grupo de Investigación de Integridad Estructural (GIE) de la Universidad de Burgos, y Coordinador de la UBU del Máster Interuniversitario. Es autor de más de 20 publicaciones científicas. Es investigador principal en varios proyectos de investigación del plan nacional y de proyectos regionales, así como de numerosos convenios de investigación con empresas del campo industrial. 